\title{
Caracterização da fusão caruncular em gestações naturais e de conceptos bovinos clonados ${ }^{1}$
}

\author{
Rodrigo da Silva Nunes Barreto ${ }^{2}$, Maria Angélica Miglino ${ }^{3}$ Flávio Vieira \\ Meirelles ${ }^{4}$, José Antônio Visintin ${ }^{5}$, Silmara Marcolino da Silva ${ }^{2}$, Kelen Cristina \\ Burioli $^{2}$, Ricardo da Fonseca², Cláudia Bertan², Antonio Chaves de Assis \\ Neto $^{2}$ e Flávia Thomaz Verechia Pereira ${ }^{2 *}$
}

\begin{abstract}
Barreto R.S.N., Miglino M.A., Meirelles F.V., Visintin J.A., Silva S.M., Burioli K.C., Fonseca R., Bertan C., Assis Neto A.C. \& Pereira F.T.V. 2009. [Characterization of the caruncular fusion in gestations of natural and cloned bovine conceptuses.] Caracterização da fusão caruncular em gestações naturais e de conceptos bovinos clonados. Pesquisa Veterinária Brasileira 29(10):779-787. Laboratório de Morfofisiologia da Placenta e Embrião, Faculdade de Zootecnia, Universidade Estadual Paulista, SP294 Km 651, Dracena, SP 17900-000, Brazil. E-mail: fverechia@dracena.unesp.br

The objective of the study was to compare the characteristics of the caruncular fusion in gestations of non-cloned and cloned conceptuses. The non-cloned conceptuses were divided according to the gestation period: Group I ( 2 to 3 months; $n=9$ ), II (4 to $6 ; n=9$ ); III ( 7 to $8 ; n=10)$ and IV $(9 n=7)$. The cloned conceptuses formed the Group V: 9 months; $\mathrm{n}=4$. The caruncles were observed macroscopically (number and dimensions: length, width and height), microscopically and submitted to statistical analysis (5\% of significance). We observed three types of macroscopic caruncular fusions: oval (morphologically normal); two united adjacent caruncles and the lobulated type, characterized by regions with several united caruncles presenting a false fusion or deformation of the caruncular parenchyma. The length of the caruncles was $1.55 \pm 0.57 ; 2.45 \pm 0.55 ; 4.66 \pm 2.0$ and $5.72 \pm 1.90 \mathrm{~cm}$ for the groups I, II, III, IV respectively. As for the height, the caruncles presented a lineal growth during the gestation: $0.40 \pm 0.15 ; 0.57 \pm 0.21 ; 1.0 \pm 0.48$ and $1.80 \pm 0.91 \mathrm{~cm}$, for the respective groups I, II, III and IV. The width of the caruncles was similar between the groups I and II $(0.97 \pm 0.30$ e $1.42 \pm 0.71 \mathrm{~cm})$ and the groups III and IV $(2.68 \pm 1.22$ and $3.52 \pm 1.16 \mathrm{~cm})$. When the group $V$ was compared to the IV, the caruncles of the group $V$ presented a larger length $(5.72 \pm 1.90 \mathrm{vs} .7 .88 \pm .13 \mathrm{~cm})$ and width $(3.52 \pm 1.16$ vs. $4.93 \pm 1.46 \mathrm{~cm})$, however they were similar in height $(1.80 \pm 0.91$ and $2.25 \pm 0.67 \mathrm{~cm})$. We verified that in gestations of cloned conceptuses the caruncles presented a larger development than in gestations of non-cloned conceptuses. The fusioned caruncles presented measurements statistically similar to the isolated ones in all the parameters and groups. Under light microscopy, we observed the formation of a stromal axis from the basis of the caruncle to the apex of the fusional fissure, with the histological constitution
\end{abstract}

\footnotetext{
${ }^{1}$ Recebido em 22 de setembro de 2008.

Aceito para publicação em 16 de abril de 2009.

2 Laboratório de Morfofisiologia da Placenta e Embrião, Faculdade de Zootecnia, Universidade Estadual Paulista (Unesp), Rodov. Cmte João Ribeiro de Barros (SP294), Km 651, Dracena, SP 17900-000, Brasil. *Autor para correspondência: fverechia@dracena.unesp.br

${ }^{3}$ Departamento de Cirurgia, Faculdade de Medicina Veterinária e Zootecnia (FMVZ), Universidade de São Paulo (USP), Av. Prof. Dr. Orlando Marques de Paiva 87, São Paulo, SP 05508-270, Brasil.

${ }^{4}$ Laboratório de Morfologia Molecular e Desenvolvimento (LMMD),
}

Faculdade de Zootecnia e Engenharia de Alimentos (FZEA), USP, Campus de Pirassununga, Av. Duque de Caxias Norte 225, Rodov. Anhangüera, Pirassununga, SP 13635-900, Brasil.

${ }^{5}$ Departamento de Reprodução Animal, FMVZ-USP, São Paulo, SP.

${ }^{6}$ Histosec Embedding Agent, Merck KGaA, Darmstadt, Germany.

7 Hydroxyethyl methacrylate, Historesin, Leica, LKB 2218-500, Sweden.

${ }^{8}$ R Development Core Team (2006). R: A language and environment for statistical computing. R Foundation for Statistical Computing, Vienna, Austria. ISBN 3-900051-07-0, URL http://www.R-project.org 
similar to the endometrial stroma. Three microscopic shapes were also unpublished defined: true fusion with a single axis evident below the fusional fissure; pseudofusion with a double axis in "H" shape and false fusion with absence of the axis. The first two formats were associated to the oval and lobulated caruncles and the last one to the false fusion with deformation of the caruncle parenchyma. The fusional axis increased in size along the gestation among the groups I, II, III and IV. The group V presented a larger length and width of the axis when compared to the group IV. Thus, in gestations of cloned conceptuses a destruction of the lateral epithelium of the caruncles is associated to an incompetence in the maternal-fetal interdigitation, that compromises the cotyledonary fusion. We suggest that, in gestations derived of cloned conceptuses, the increase of the size of the caruncular fusions is possibly associated to a compensatory mechanism for the metabolic exchanges between mother and fetus, in reason of the smallest number of isolated caruncles.

INDEX TERMS: Caruncular fusion, gestation, cloned cattle.

RESUMO.- O objetivo deste trabalho foi comparar as fusões carunculares em gestações de conceptos não clonados (CNC) e conceptos clonados (CC). Os CNC foram divididos segundo o período de gestação em Grupo I (2-3 meses, $n=9$ ), Grupo II (4-6 meses, $n=9)$, Grupo III (7-8 meses, $n=10$ ) e Grupo IV (9 meses, $n=7)$. Os CC formaram o Grupo V (9 meses, $n=4)$. As carúnculas foram observadas macroscopicamente (número e dimensões: comprimento, largura e altura), microscopicamente e submetidas à análise estatística (5\% de significância). Observaram-se três tipos de fusões carunculares macroscópicas: ovais (morfologicamente normais); duas carúnculas adjacentes unidas e do tipo lobuladas, caracterizadas por regiões com várias carúnculas unidas apresentando falsa fusão ou deformação do parênquima caruncular. O comprimento das carúnculas foi de 1,55 $\pm 0,57$; $2,45 \pm 0,55 ; 4,66 \pm 2,00$ e $5,72 \pm 1,90 \mathrm{~cm}$ para os Grupos I, II, III e IV, respectivamente. Quanto à altura, as carúnculas apresentaram um crescimento linear durante a gestação e foram de $0,40 \pm 0,15 ; 0 ; 57 \pm 0,21 ; 1,00 \pm 0,48$ e 1,80 $\pm 0,91 \mathrm{~cm}$, para os respectivos Grupos I, II, III e IV. A largura das carúnculas foi semelhante entre os Grupos I e II $(0,97 \pm 0,30$ e 1,42 $\pm 0,71 \mathrm{~cm})$ e os Grupos III e IV $(2,68 \pm 1,22$ e 3,52 $\pm 1,16 \mathrm{~cm})$. Quando o Grupo V foi comparado ao Grupo IV, as carúnculas do Grupo $\mathrm{V}$ apresentaram maior comprimento $(5,72 \pm 1,90$ vs. $7,88 \pm 2,13 \mathrm{~cm})$ e largura $(3,52 \pm 1,16$ vs. $4,93 \pm 1,46 \mathrm{~cm})$, porém foram semelhantes em altura $(1,80 \pm 0,91 \mathrm{e}$ $2,25 \pm 0,67 \mathrm{~cm})$. Verificou-se que em gestações de CC, as carúnculas apresentaram maior desenvolvimento que em gestações de CNC. As carúnculas fusionadas apresentaram medidas estatisticamente semelhantes às isoladas em todos os parâmetros e grupos. Sob microscopia de luz, observou-se a formação de um eixo estromal, da base da carúncula ao ápice da fissura fusional, de constituição histológica semelhante ao estroma endometrial. Também foram ineditamente definidos três formatos microscópicos: fusão propriamente dita com eixo único, evidente abaixo da fissura fusional; pseudofusão com eixo duplo em forma de "H" e a falsa fusão com ausência do eixo. Os dois primeiros formatos referem-se às carúnculas ovais e lobuladas e o último à falsa fusão com deformação do parênquima caruncular. O eixo fusional aumentou de tamanho durante a gestação entre os Grupos I, II, III e IV. O Grupo V apresen- tou maior comprimento e largura de eixo quando comparado ao Grupo IV. Sendo assim, em gestações de CC a destruição do epitélio lateral das carúnculas está associada a uma incompetência na interdigitação materno-fetal, que comprometem a fusão cotiledonária. Sugere-se que, em gestações provenientes de CC, o aumento do tamanho das fusões carunculares possivelmente associa-se a um mecanismo compensatório para as trocas metabólicas entre mãe e feto, em razão do menor número de carúnculas isoladas.

TERMOS DE INDEXAÇÃO: Fusão caruncular, gestação, clones bovinos.

\section{INTRODUÇÃO}

A placenta cotiledonária, típica de ruminantes, é caracterizada por muitas regiões placentárias (placentônios) que estão separadas por áreas intercotiledonárias de cório liso (Leiser \& Kaufmann 1994). Os placentônios são compostos por uma parte fetal, o cotilédone, e uma parte materna, a carúncula (Leiser et al 1998).

De acordo com Abdel-Raouf \& Badavi (1966), as carúnculas foram assim nomeadas por Burckhardt em 1834. Elas são áreas especializadas não glandulares, e são essencialmente locais de extenso tecido conjuntivo denso subepitelial, e em outros locais, como uma fina camada imediatamente abaixo do epitélio. São moderadamente vascularizadas, e seus pequenos vasos sangüíneos tendem, em conjunto, seguir em direção à superfície (Amoroso 1952).

Durante o inicio da gestação, o epitélio entre a região caruncular e intercaruncular, depois da fixação do cório com suas interdigitações microvilosas formam a placenta. Cada carúncula subseqüentemente cresce rapidamente e desenvolve criptas para aumentar a área de superfície de contato. A conexão do vilo coriônico na cripta caruncular serve para manter a aposição física completa da placenta. A maior importância das carúnculas, é que elas desenvolvem uma extensa cama vascular e são o principal local de trocas de gases e pequenas moléculas (Atkinson et al.1984).

Björkman $(1954,1969)$ descreve que a placenta bovina está completamente desenvolvida com 170 dias de 
gestação, e a partir desse período o crescimento é lento e ocorre principalmente na periferia do placentônio, dando a forma de cogumelo.

Hilty (1908) relata que a variação do tamanho das carúnculas depende do estágio de gravidez, assim como de sua posição no útero. As carúnculas do corno uterino não gravídico medem 6,0 x 3,5mm, com uma altura de 2,5$5 \mathrm{~mm}$. E no corno gravídico, o máximo que as carúnculas puderam chegar na $6^{a}$ semana de gestação foi $9 \times 4 \times$ $3 \mathrm{~mm}$ e $78 \times 34 \times 26 \mathrm{~mm}$ na $26^{\mathrm{a}}$ semana. E ainda adicionou que, 43 dias após o parto, a medida máxima da carúncula é $10 \times 4$ x 3mm. Zieger \& Zschiesche (1922) relatam em sua pesquisa uma carúncula medindo $25 \times 9 \times 4 \mathrm{~cm}$, mas explicam que isso é a fusão entre algumas carúnculas.

Em gestações de conceptos clonados, os tamanhos e números de placentônios, sofrem variações, sendo maiores e menos numerosos quando comparados aos de não clonados, porém são encontradas diversidades na literatura. Leiser et al. (1998) afirma que são encontrados 89 placentônios em gestações naturais, Miles et al. (2004) encontraram 81,3 e Bertolini \& Anderson (2002) cerca de 102; para as gestações de conceptos clonados, Bertolini e Anderson (2002) observaram 81, Pereira et al. (2003) 39 e Miglino et al. (2007) 85,8 placentônios (com variação de 20 a 137).

Miglino et al. (2007) relatou a ocorrência de grandes placentônios e menores quantidades, dos mesmos, nas oito gestações, a termo, de conceptos bovinos clonados analisadas. Também observou a união de pequenos placentônios, provavelmente por crescimento extremo da sua margem, levando a fusão de várias carúnculas, sendo que em algumas era visível um corte separando-as.

Além destas, existem muitas outras deformações na placentação de clones tais como, hidroalantóide, hidroâmnio, síndrome do bezerro grande, hipovascularização, hemorragias, edema de membranas fetais, etc. Por todos estes motivos a clonagem animal por transferência nuclear ainda é um procedimento altamente ineficiente em todas as espécies em que foi realizada, devido a altas taxas de abortos durante a gestação e morte pós-natal (De Souza et al. 2001).

Apesar de existirem, atualmente, vários relatos de diversos autores, sobre anomalias placentárias relacionados ao insucesso da clonagem e comparados a gestações naturais, porém a existência ou mensuração de dados sobre fusão caruncular em animais naturais não é citada em nenhum trabalho conhecido, apenas são citadas prováveis fusões, e ainda quando se trata de comparação com animais clonados a literatura ainda é mais escassa, por isso observações comparativas entre animais naturais em várias idades gestacionais com gestações de conceptos clonados, a termo, serão descritas neste trabalho.

\section{MATERIAL E MÉTODOS}

Foram utilizados 63 úteros de vacas, azebuadas com gestações oriundas de monta natural ou inseminação artificial, obtidos em abatedouros frigoríficos da região de Dracena (SP) e Presidente Prudente (SP). Foram divididos por idade de gestação: Grupo I, fêmeas com 31-90 dias de prenhez $(n=17)$; Grupo II, 91-180 dias ( $n=21)$; Grupo III, 181-240 dias $(n=14)$; e Grupo IV, 241 280 dias $(n=11)$.

Além das amostras supracitadas foram utilizados mais 4 úteros de vacas azebuadas, com gestação de conceptos clonados por transferência nuclear de células somáticas adultas e fetais, procedimento realizado nos laboratórios do Prof. Dr. José A. Visintin (Faculdade de Medicina Veterinária e Zootecnia, USP) e do Prof. Dr. Flávio Vieira Meirelles (Faculdade de Zootecnia e Engenharia de Alimentos, USP). As gestações chegaram a termo com 290 dias, em média, e as amostras foram obtidas junto ao laboratório da Profa Dra Maria Angélica Miglino (FMVZ-USP). Estes animais compõem o Grupo V.

Após o sacrifício houve a inversão do útero, desconexão carúncula-cotiledonária e análise segundo a presença de fusão caruncular, com posterior mensurações de todas as carúnculas (apenas nas gestações com fusão caruncular) em quantidade e comprimento, largura e altura (em centímetros, com auxílio de um paquímetro) e foram fotodocumentadas.

A região das fissuras fusionais foi recortada e fixada, com solução aquosa de formaldeído a $10 \%$ em tampão fosfato (Dulbecco's phosfate buffer saline, DPBS, Gibco Co., USA) 0,1M $\mathrm{pH} 7,4$ ou em solução aquosa de paraformoldeído a $4 \%$ em mesmo tampão, e incluídos em Histosec ${ }^{6}$ ou Historesina ${ }^{7}$. Em seguida foram obtidos cortes que foram corados rotineiramente por Hematoxilina de Harris - Eosina (HE), Tricromo de Masson, Picrosirius, reação histoquímica de PAS (Periodic Acid-Schiff) contracorada com Hematoxilina de Harris, Azul de Toluidina, Azul de Metileno-Fucsina básica e Hematoxilina-Floxina.

Em ambos processos as amostras foram analisadas em microscópio óptico Leica DM 2500, fotomicrografados com auxilio de câmera Leica DFC 300 FX, acoplada no microscópio citado e conectada em microcomputador, em seguida os eixos fusionais foram mensurados, em $\mu \mathrm{m}$, com auxilio do programa Qwin (versão V3.3.1).

Todos os dados foram inseridos em planilhas de cálculo e submetidos à análise no programa estatístico $R^{8}$ (versão 2.3.0), sob testes de hipótese (T e Qui-quadrado).

\section{RESULTADOS E DISCUSSÃO}

Dos 63 úteros gestantes de conceptos não clonados (CNC, Grupos I-IV) coletados, 35 apresentaram fusões, mostrando que $55,6 \%$ das gestações apresentaram este tipo de estrutura, desde períodos gestacionais mais iniciais (69 dias) a outros mais tardios (275 dias). As quatro amostras de conceptos clonados (CC, Grupo V) apresentaram fusões carunculares, mostrando uma freqüência maior que nos CNC de mesma idade (Grupo IV), 72,7\% (Quadro 1).

\section{Quadro 1. Amostras com presença e ausência de fusão} caruncular nos grupos de CNC. Dracena, 2008

\begin{tabular}{lccccc}
\hline & \multicolumn{5}{c}{ Úteros de CNC } \\
\cline { 2 - 6 } & Presença & $\%$ & Ausência & $\%$ & Total \\
\hline Grupo I & 9 & 52,9 & 8 & 47,1 & 17 \\
Grupo II & 9 & 42,9 & 12 & 57,1 & 21 \\
Grupo III & 9 & 64,3 & 5 & 35,7 & 14 \\
Grupo IV & 8 & 72,7 & 3 & 27,3 & 11 \\
Total & 35 & 55,6 & 28 & 44,4 & 63
\end{tabular}


Talvez porque os placentônios em gestações de CC têm crescimento vigoroso até o parto, segundo Bertolini \& Anderson (2002) e não como nos CNC até aos 170 dias gestações (Björkman 1954).

As fusões carunculares, em todos os grupos, estavam em sua maioria dispostas na linha antimesometrial no segmento médio do corno uterino, sendo também encontradas proximais a tuba uterina e outras proximais ao cor- po do útero, sempre isoladas umas das outras. Eram de fácil visualização, pois mesmo antes da desconexão cotilédone-carúncula a fissura fusional se mostrava evidente (Fig.1a,b).

Foram encontradas fusões carunculares que variaram em tamanho desde $0,6 \times 0,5 \times 0,4 \mathrm{~cm}$ a $10,8 \times 5,4 \times 2,6 \mathrm{~cm}$ nos grupos de CNC. Valores semelhantes ao encontrado por Hilty (1908) $0,9 \times 0,4 \times 0,3 \mathrm{~cm}$ a $7,8 \times 3,4 \times 2,6 \mathrm{~cm}$.

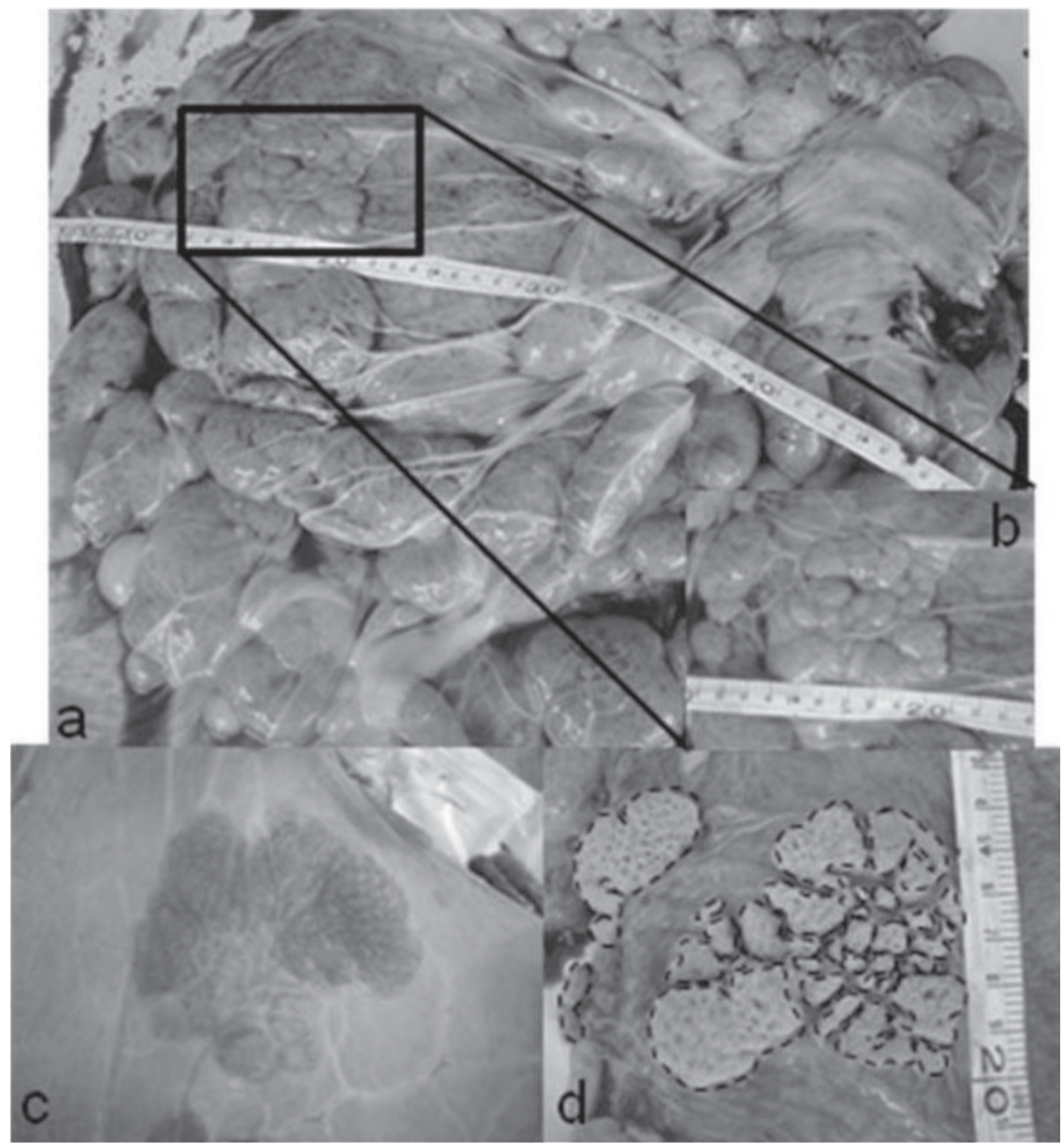

Fig.1. Útero bovino gestante de CNC com 182 dias e detalhes da fusão caruncular antes e após desconexão carúncula-cotiledonária. (a) Útero aberto na linha antimesometrial; (b) fusão caruncular antes da desconexão; (c) cotilédone após desconexão, sem indicio de fusão cotiledonária; (d) fusão caruncular, após desconexão, formada por várias carúnculas (círculos). 
Quadro 2. Médias e desvios-padrão das variáveis macroscópicas de comprimento, largura e altura das carúnculas nos diversos grupos. Dracena, 2008

\begin{tabular}{|c|c|c|c|c|c|c|}
\hline & \multicolumn{2}{|c|}{ Comprimento } & \multicolumn{2}{|c|}{ Largura } & \multicolumn{2}{|c|}{ Altura } \\
\hline & Fusionadas & Avulsas & Fusionadas & Avulsas & Fusionadas & Avulsas \\
\hline Grupo I & $1,55 \pm 0,57^{\mathrm{Aa}}$ & $1,27 \pm 0,52^{\mathrm{Aa}}$ & $0,97 \pm 0,30^{\mathrm{Aa}}$ & $0,86 \pm 0,57^{\mathrm{Aa}}$ & $0,40 \pm 0,15^{\mathrm{Aa}}$ & $0,41 \pm 0,18^{\mathrm{Aa}}$ \\
\hline Grupo II & $2,45 \pm 0,55^{\mathrm{Aa}}$ & $2,12 \pm 0,94^{\mathrm{Ba}}$ & $1,42 \pm 0,71 \mathrm{Ba}$ & $1,45 \pm 0,66^{\mathrm{Ba}}$ & $0,57 \pm 0,21^{\mathrm{Ba}}$ & $0,69 \pm 0,56^{\mathrm{Bb}}$ \\
\hline Grupo III & $4,66 \pm 2,41^{\mathrm{Ba}}$ & $4,59 \pm 1,82^{\mathrm{Cb}}$ & $2,68 \pm 1,22^{\mathrm{Ca}}$ & $3,09 \pm 1,30^{\mathrm{Ca}}$ & $1,00 \pm 0,48^{\mathrm{Ca}}$ & $1,33 \pm 0,54^{\mathrm{Ba}}$ \\
\hline Grupo IV & $5,72 \pm 1,90^{\mathrm{Ba}}$ & $4,84 \pm 1,93^{\mathrm{Da}}$ & $3,52 \pm 1,16^{\mathrm{Ca}}$ & $3,25 \pm 1,36^{\mathrm{Da}}$ & $1,80 \pm 0,91 \mathrm{Da}$ & $1,51 \pm 0,63^{\mathrm{Ab}}$ \\
\hline Grupo V & $7,88 \pm 2,13^{\mathrm{Ba}}$ & $6,00 \pm 2,73^{\mathrm{Ea}}$ & $4,93 \pm 1,46 \mathrm{Ca}$ & $3,92 \pm 1,89 \mathrm{Ea}$ & $2,25 \pm 0,67^{\mathrm{Da}}$ & $1,86 \pm 0,85^{\mathrm{Aa}}$ \\
\hline
\end{tabular}

Teste $\mathrm{F}$ ao nível de $5 \%$ de significância. Letras maiúsculas indicam igualdade na vertical e minúsculas na horizontal.

Quadro 3. Distribuição do comprimento das carúnculas. Dracena, 2008

\begin{tabular}{lccccccccccccc}
\hline & \multicolumn{10}{c}{ Distribuição (em \%) } & \multicolumn{10}{c}{ comprimento $(\mathrm{em} \mathrm{cm})$} & das carúnculas \\
& \cline { 2 - 15 }$y, 0$ & 1,0 & 2,0 & 3,0 & 4,0 & 5,0 & 6,0 & 7,0 & 8,0 & 9,0 & 10,0 & 11,0 & 12,0 \\
\hline Grupo I & 37,67 & 52,74 & 9,59 & 0,00 & 0,00 & 0,00 & 0,00 & 0,00 & 0,00 & 0,00 & 0,00 & 0,00 & 0,00 \\
Grupo II & 5,71 & 36,76 & 14,47 & 12,33 & 4,57 & 4,59 & 1,14 & 0,91 & 0,23 & 0,46 & 0,00 & 0,00 & 0,00 \\
Grupo III & 0,00 & 4,11 & 14,38 & 16,67 & 24,66 & 22,34 & 12,10 & 5,94 & 3,88 & 1,14 & 0,23 & 0,23 & 0,00 \\
Grupo IV & 0,92 & 1,36 & 11,96 & 18,10 & 18,10 & 15,61 & 14,72 & 8,59 & 3,07 & 0,61 & 0,61 & 0,31 & 0,00 \\
Grupo V & 1,02 & 6,12 & 9,18 & 9,18 & 13,77 & 10,20 & 13,26 & 11,72 & 12,24 & 4,59 & 5,10 & 3,06 & 1,02
\end{tabular}

Nos CC, estes valores variaram de 4,6 $\times 2,6 \times 1,3$ a $12,2 \times 6,7 \times 2,1 \mathrm{~cm}$, sendo maiores que $62,7 \%$ das carúnculas avulsas (carúnculas não fusionadas), valores maiores que o grupo de CNC de idade semelhante (Grupo IV), onde houve variação de $1,0 \times 1,1 \times 0,6$ a 10,8 × 5,4 ×2,6cm, maiores que $32,34 \%$ das isoladas (Quadros 2 e 3 ).

O número de fusões em cada gestação variou de um a oito, sendo que não houve relação com sexo do feto, mas alguma influência sobre a idade, pois os animais com mais de 90 dias de gestação obtiveram os maiores números, porém menor porcentagem em relação às carúnculas totais. A quantidade de fusões carunculares por gestação no Grupo V variou de duas a oito quando no Grupo IV variou de duas a sete, porém a porcentagem de fusões nos $\mathrm{CC}$ em relação às carúnculas totais é três vezes maior que nos CNC, visto que a quantidade de carúnculas totais é quase a metade.

Em um dos animais do Grupo V, houve a presença de hidroalantóide, o que levou o feto e a vaca à morte, e, após a abertura e dissecação do útero, foi observada a presença de carúnculas gigantes e número reduzido dos mesmos, cerca de 39, devido a ocorrência de áreas com agenesia de carúnculas.

Como visto acima, a diferença básica entre as gestações de conceptos clonados (CC) e de conceptos não clonados (CNC), foram em tamanho e quantidades de carúnculas, assim como afirmam Bertolini e Anderson (2002) e Miglino et al. (2007).

Após a desconexão cotilédone-carúncula puderam-se visualizar claramente as áreas fusionadas das carúnculas, e aspecto "favo-de-mel" das criptas carunculares, e o cotilédone íntegro, ou seja, este não apresenta nenhum indicio de fusão cotilédone-cotiledonária (Fig.1c,d).

Com isso, pode-se dizer que a fusão caruncular ocorre antes da fixação cotilédone-carúncula, mas não antes dos primeiros contatos entre as duas estruturas que ocor- rem por volta do $20^{\circ}$ dia de gestação (King \& Atkinson 1987 ), pois as maiores quantidades de fusões acontecem após os 90dias.

As fissuras fusionais puderam ser observadas únicas ou em par, de um lado ao outro ou do centro para periferia, mas sempre se dirigiam da superfície da carúncula em direção ao pedúnculo sendo modelada pelo cotilédone, dando assim forma à fusão.

Os formatos macroscópicos das fusões carunculares observadas foram três: oval, onde se visualizava duas carúnculas vizinhas justapostas, bastante comum as amostras dos grupos de CNC (Fig.2a,e); lobulada, região com a justaposição de várias carúnculas, mais comuns aos CC (Fig.2b,f); e a falsa fusão, subdivida em dois tipos, aquela apenas com depressão no parênquima de uma carúncula dando a impressão de uma falsa fissura, não observada em CC (Fig.2c) e regiões de carúnculas semelhantes ao formato lobulado, porém após observação minuciosa, podemos observar estreitas faixas de tecido intercaruncular separando as carúnculas (Fig.2d,g).

A existência do formato oval foi citada por Marques et al (2007) onde afirma que a fusão de carúnculas vizinhas forma placentônios maiores. O formato lobulado concorda com um dos formatos de placentônios encontrados Zieger \& Zschiesche (1922), onde eles encontraram algo que aparentemente era o resultado da fusão entre carúnculas vizinhas. Dados também encontrados por Abdel-Raouf \& Badavi (1966). A falsa fusão, subtipo com faixas intercarunculares separando as carúnculas, também foi encontrado por Miglino et al. (2007), porém não foi nomeado desta forma.

Para comprimento de carúnculas vimos que existe uma igualdade entre as fusionadas e isoladas, exceto para o Grupo III, vimos também que nas fusionadas temos igualdade entre os Grupos I e II, como também para os Grupos III e IV, já para isoladas os valores variaram em todos os grupos (Quadro 2). 

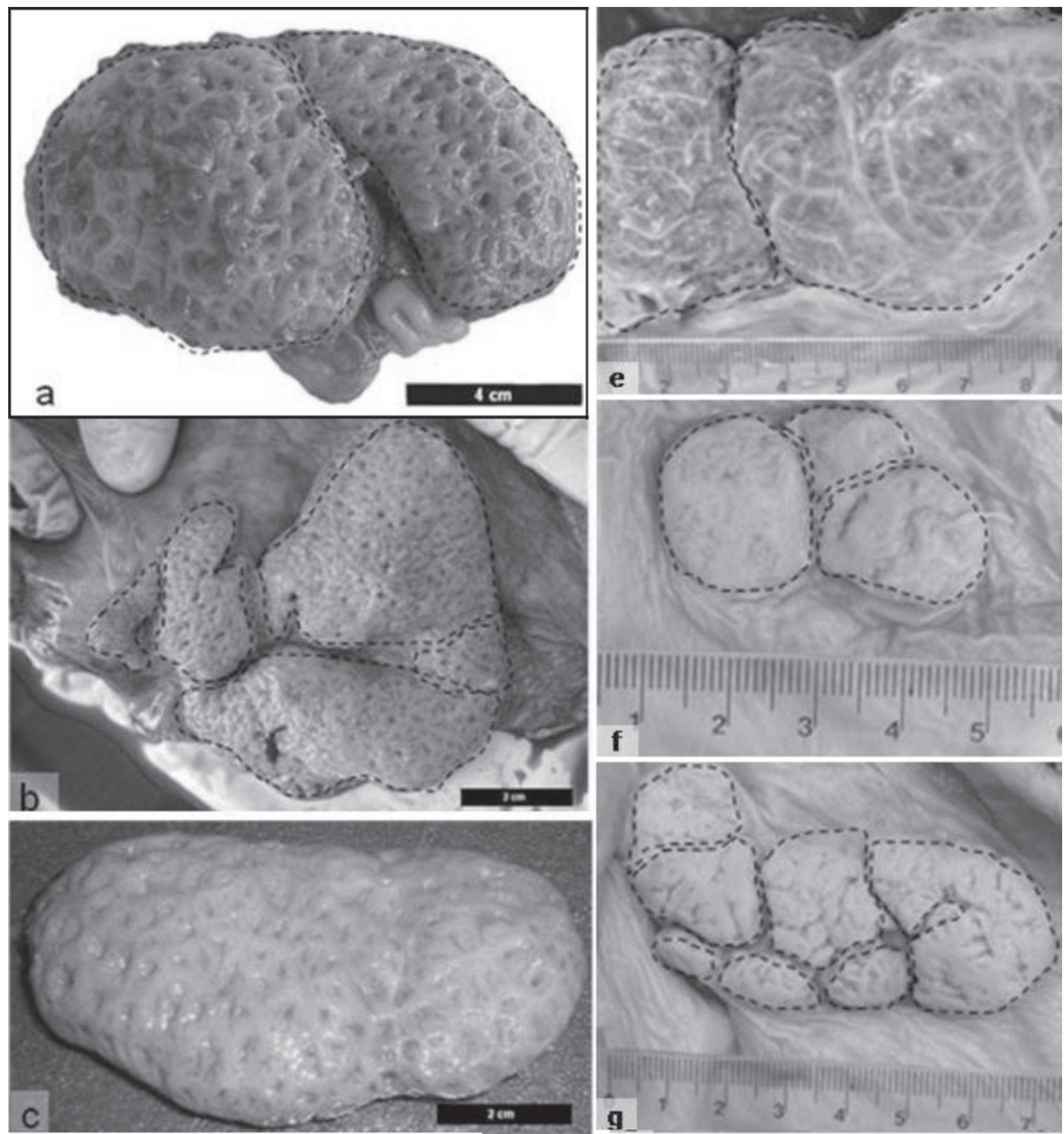

Fig.2. Formatos macroscópicos da fusão caruncular em CNC (esquerda) e CC (direita). Fusão oval $(\mathbf{a}, \mathbf{e})$, lobulada $(\mathbf{b}, \mathbf{f})$, falsa fusão por depressão do parênquima (c) e falsa fusão com faixa intercaruncular $(\mathbf{d}, \mathbf{g})$.

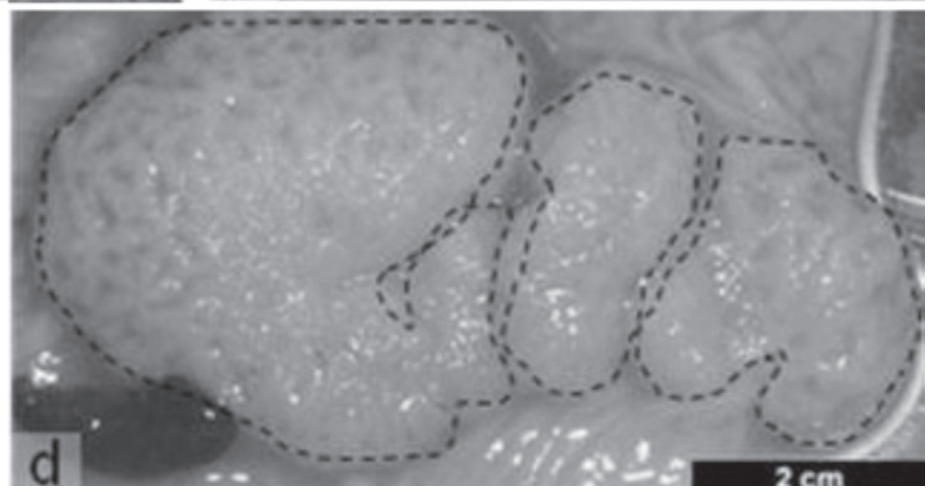



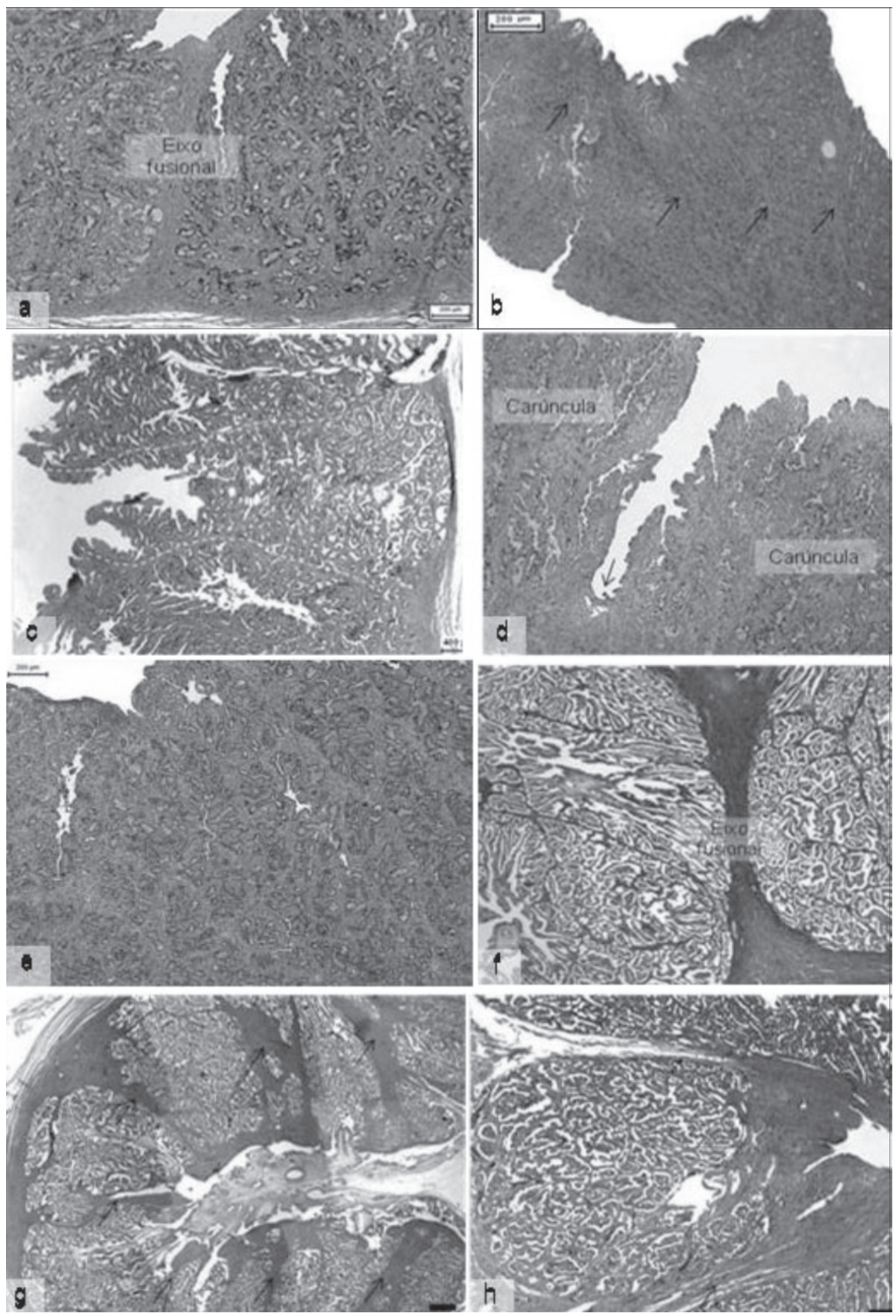

Fig.3. Formatos microscópicos da fusão caruncular em CNC (a,b,c, d,e e) e CC (f, $, \mathbf{g}, \mathbf{h})$. Fusão caruncular propriamente dita (a,f), radial $(\mathbf{b}, \mathbf{g})$, pseudofusão $(\mathbf{c}, \mathbf{h})$, falsa fusão com faixa intercaruncular $(\mathbf{d})$ e falsa fusão com depressão do parênquima (e). Setas $=$ eixo fusional de tecido conjuntivo denso não modelado; a,b,d,e: barra $=200 \mu \mathrm{m} ; \mathrm{c}, \mathrm{f}, \mathrm{h}$ : barra $=400 \mu \mathrm{m} ; \mathrm{g}$ : barra $=130 \mu \mathrm{m}$ 
Na variável largura, temos igualdade total entre os dois tipos de carúnculas (fusionadas e isoladas), uma igualdade para os Grupos III e IV das fusionadas e diferença entre as isoladas, assim como para a variável comprimento (Quadro 2).

Analisando a altura das carúnculas temos diferenças de tamanhos, entre os dois tipos, nos Grupos II e IV, e entre grupos houve igualdade apenas para as isoladas nos Grupos I e IV, como nos II e III (Quadro 2).

Comparando Grupo V com Grupo IV, temos que as medidas de comprimento, largura e altura nas carúnculas fusionadas e nas isoladas foram sempre maiores, numericamente, no Grupo V, porém estatisticamente houve diferença apenas para comprimento e largura nas isoladas (Quadro 2).

Vimos ainda que em relação à distribuição dos valores de comprimento para carúnculas totais, há um crescimento constante sendo que no primeiro grupo esses valores não ultrapassam a 2,0cm, enquanto que o Grupo II alcança $9,0 \mathrm{~cm}$ e os Grupos III e IV $11,0 \mathrm{~cm}$. Porém vemos que mesmo com o aumento de tamanho temos uma distribuição normal dos valores, ou seja, os valores extremos possuem menor porcentagem que os valores medianos em todos os grupos (Quadro 3).

Sob microscopia de luz, as fusões carunculares foram caracterizadas por um eixo perpendicular ao pedúnculo caruncular, e se estendia, sem ramificações significantes, contornando a fissura, e seguindo em direção ao pedúnculo caruncular. Observamos ainda o estroma endometrial criptal e o peduncular, idênticos histologicamente ao estroma endometrial fusional, ou seja, constituído de tecido conjuntivo denso não-modelado, rico em fibras colágenas.

Células gigantes trofoblásticas binucleadas provenientes da migração de células do epitélio fetal (trofoblasto), puderam ser observadas no eixo fusional durante as várias fases da gestação, bem como no estroma endometrial e epitélio criptal. Pôde-se observar claramente a diferença de coloração das células fetais e maternas, onde as células fetais apresentavam-se mais fortemente coradas. Também foi observado que as células gigantes trofoblásticas binucleadas que se encontram no estroma fusional, apresentavam núcleos com a cromatina frouxa.

Os tipos de fusões foram classificados em quatro aspectos de acordo com a sua morfologia microscópica: fusão propriamente dita, onde o eixo fusional encontra-se logo abaixo a fissura (Fig.3a,f); radial, surgimento de eixos de vários pontos do pedúnculo e tocando também vários pontos da fissura (Fig.3b,g); pseudofusão possui dois eixos fusionais estreitos e paralelos à fissura seguindo em direção ao estroma peduncular interligando-se e formando um "H" (Fig.3c,h); e a falsa fusão, com ausência de eixo fusional, ou por haver uma estreita faixa de tecido intercaruncular separando as carúnculas (Fig.3d) ou por existir apenas uma depressão no parênquima (Fig.3e).

Analisando as quantidades de cada formato microscópico de fusão, vimos que não há o predomínio de um único
Quadro 4. Porcentagem dos tipos microscópicos de fusão caruncular em cada grupo. Dracena, 2008

\begin{tabular}{lcccc}
\hline & \multicolumn{4}{c}{ Tipos microscópicos de fusão caruncular } \\
\cline { 2 - 5 } & Propriamente dita & Radial & Pseudofusão & Falsa \\
\hline Grupo I & 50,00 & 0 & 18,75 & 31,25 \\
Grupo II & 31,82 & 13,64 & 13,64 & 40,91 \\
Grupo III & 12,50 & 0 & 37,50 & 50,00 \\
Grupo IV & 20,00 & 30,00 & 30,00 & 20,00 \\
Grupo V & 21,43 & 14,29 & 57,14 & 7,14
\end{tabular}

tipo nos diversos grupos, ocorrendo então uma oscilação, tal fato pode estar relacionado ao crescimento, em número, e organização das fusões carunculares (Quadro 4).

Os formatos microscópicos (fusão propriamente dita, radial e pseudofusão) estão relacionados aos formatos macroscópicos oval e lobulado, e a falsa fusão (microscópico), relaciona-se com os dois subtipos da falsa fusão (macroscópico).

Visualmente, observamos um aumento em comprimento e largura no eixo fusional, com o avançar da gestação, fato que também ocorre com os vasos sangüíneos do eixo que aumentam seu calibre.

Porém foi possível mensurar apenas os eixos das fusões propriamente dita e pseudofusão, visto a heterogeneidade da fusão radial e a ausência de eixos na falsa fusão. Para o primeiro formato, foi mensurado comprimento, largura no ápice, no meio e na base do eixo. Para o segundo formato mediu-se a profundidade da fusão e largura dos dois eixos paralelos.

Para fusão propriamente dita, temos um crescimento numérico visível em todas medidas, porém houve diferença estatística apenas no Grupo IV para comprimento e entre os Grupos I e II com os Grupos III e IV para largura no ápice. Entre os Grupos IV e V, obtivemos grandes variações numéricas, porém diferenças estatísticas ocorreram apenas para comprimento de eixo.

Na pseudofusão também ocorre um crescimento numérico, com um pequeno decréscimo no Grupo III para ambas larguras, porém estatisticamente houve diferença significativa apenas nos Grupos I e II, para a variável profundidade, sendo que o Grupo II foi semelhante aos Grupos III e IV. Entre os Grupos IV e V não houve diferença.

Através de todos os dados obtidos, podemos sugerir que o processo de fusão caruncular poderia ser por aproximação entre carúnculas vizinhas, devido ao crescimento de carúnculas existentes ou pelo surgimento de novas. Após a aproximação total das carúnculas ocorria destruição do epitélio e unificação da estreita camada de estroma endometrial sub-epitelial.

\section{CONCLUSÕES}

Pelo fato de não ocorrer fusão cotilédone-cotiledonária e o cotilédone se moldar na fissura fusional, podemos sugerir que a fusão caruncular ocorre antes da total fixação dos vilos cotiledonários nas criptas carunculares.

Segundo a sugestão da formação da fusão caruncular seria possível afirmar que a falsa fusão com carúnculas 
próximas, porém separadas por estreita faixa de tecido intercaruncular, é um estágio de formação da fusão caruncular.

O formato microscópico de fusão radial possui extensa área de tecido conjuntivo, em vista da quantidade de eixos fusionais que são formados, principalmente nas gestações de CC, tal desenvolvimento deve ocorrer na tentativa de aumentar o volume de tecido na interface materno-fetal, porém ocorre de forma errônea, pois o tecido conjuntivo, que constitui o eixo fusional, tem a função apenas de preenchimento. Além disso, este formato de fusão poderia ser transitório ou intermediário entre os outros formatos de fusão, pois possui eixos espessos como na fusão propriamente dita e mais de um núcleo de surgimento de eixos como na pseudofusão.

Com base nos dados anteriormente descritos, podemos afirmar que ocorrem algumas variações na morfologia, e aumento da quantidade e tamanho das fusões carunculares nas gestações de CC quando comparados às de $\mathrm{CNC}$, isso pode ocorrer funcionalmente como mecanismo compensatório em relação ao menor número de carúnculas isoladas.

\section{REFERÊNCIAS}

Abdel-Raouf M. \& Badavi H.M. 1966. Morphological study of uterine caruncles in Egyptian Buffalo cows. Zentralbl. Veterinärmed. 13:252263.

Amoroso E.C. 1952. Placentation, p.127-311. In: Marshall F.H.A. (Ed.), Marshall's Physiology of Reproduction. Longmans, London.

Atkinson B.A., King G.J. \& Amoroso E.C. 1984. Development of caruncular and intercaruncular regions in the bovine endometrium. Biol. Reprod. 30:763-774.

Bertolini M. \& Anderson G.B. 2002. The placenta as a contribuitor to production of large calves. Theriogenology 57:181-187.
Björkman N. 1954. Morphological and histological studies on the bovine placenta. Acta Anatomica 221(Suppl.):1-9.

Björkman N. 1969. Light and electron microscopic studies on cellular alterations in the normal bovine placentome. Anatomica Record 163:17-30.

De Souza P.A., King T., Harkness L., Young L.E., Walker S.K. \& Wilmut I. 2001. Evalution of gestational deficiences in cloned sheep fetuses and placenta. Biol. Reprod. 65:23-30.

Hilty H. 1908. Untersuchungen über die Evolution und Involution der Uterusmucosa vom Rind. Schweiz. Arch. Tierheilkd. 50:353. (Apud Abdel-Raouf \& Badavi 1966)

King G.J. \& Atkinson B.A. 1987. The bovine intercaruncular placenta throughout gestation. Anim. Reprod. Sci. 12:241-254.

Leiser R. \& Kaufmann P. 1994. Placental structure: In a comparative aspect. Exp. Clin. Endocrinol. 102:122-134.

Leiser R., Pfarrer C., Abd-Elnaeim M. \& Dantzer V. 1998. Feto-maternal anchorage in epitheliochorial and endotheliochorial placental types studied by histology and macrovascular corrosion casts. Trophoblast Res. 12:21-39.

Marques R.S., Vulcano M., Cazerta S.M.M., Miglino M.A., Assis Neto A.C. \& Pereira F.T.V. 2007. Caracterização morfológica da região intercaruncular uterina de vacas e búfalas gestantes. Biotemas 20:103-114.

Miglino M.A., Pereira F.T.V., Visintin J.A., Garcia J.M., Meirelles F.V., Rumpf R., Ambrósio C.E., Papa P.C., Santos T.C., Carvalho A.F., Leiser R. \& Carter A.M. 2007. Placentation in cloned cattle: Structure and microvascular architecture. Theriogenology 68:604-617.

Miles J.R., Farin C.E., Rodriguez K.F., Alexander J.E. \& Farin P.W. 2004. Angiogenesis and morphometry of bovine placentas in late gestation from embryos porduced in vivo or in vitro. Biol. Reprod. 71:1919-1926.

Pereira F.T.V., Miglino M.A., Visitin J., Mello M.B., Mansano J., Yamasaki W., Ambrósio C.E., Carvalho A.F., Braga F.C., Santos J.L., Leiser R. \& Carter A.M. 2003. Placentation in cloned cattle: Microvascular architeture and structure. Placenta 24:A37.

Zieger A. \& Zschiesche M. 1922. Die Diagnose der Trächtigkeit des Rindes und der Sterilität. 2. Aufl. Walter Richter, Leipzig. 64p. (Apud Abdel-Raouf \& Badavi 1966) 